\title{
Research on Small-scale Variable Capacity Evaporative Cooling Plate System for Chips with High Heating Flux
}

\author{
Tao Wang ${ }^{1, a^{*}}$, Fang Wang ${ }^{1, b}$, Hongqun Chen ${ }^{1, c}$ \\ Junyan Ou ${ }^{1, d}$, Dawei Yu ${ }^{1, e}$, Jiawen Zhang ${ }^{1, f}$ \\ ${ }^{1}$ University of Shanghai for Science and Technology, Shanghai 200093,China \\ a1533839564@qq.com, bwang1996930@163.com, '348954900@qq.com, \\ d879505354@qq.com, ${ }^{\mathrm{e}} 150158853 @ q q . c o m,{ }^{\mathrm{f}} 1564655938 @ q q . c o m$
}

Keywords: high heating flux, chip, variable capacity, evaporative cooling plate

Abstract. This paper aims to research and analyze the heat dispersion of small variable capacity refrigeration system which is used for cooling the chips with high heating flux. The cooling system was built for researching what influence refrigerant charge will bring to the performance of the system, such as suction-exhaust pressure and temperature as well as refrigerating capacity of the system. Simulation experiment has been established to contrast the performances of system with evaporative cooling plate and water-cooled cooling plate respectively. The result shows it takes less time to make the temperature of the chip stable with the former system. Moreover, the temperature can keep at a lower level.

\section{Introduction}

With the rapid development of technology, electronic products have already been used in more and more fields, with more intelligent system and tinier shape [1]. However, when the level of integration becomes higher, it will consume more energy and the system will be less stable. As a result, variety of problems will come out. Heat dissipation has already confined the development of electronic devices. The issue of thermal failure has become more and more important [2]. In terms of electronic devices with high heating flux, forced convection, heat pipe and liquid cooling are relatively mature technologies for heat dispersion nowadays.

Hong Cao etc. [3] have researched characteristic of electronic equipment heat dissipation by force convection and confirmed the reliability of the measurement software. Anlin Zhao, Jianyun Deng etc. have researched heat pipe used for CPU heat dissipation. Different refrigerant charges and air speed was considered in the experiment [4].

Small-sized vapor compression refrigeration is based on the characteristic that refrigerant phase change absorbs so large amounts of heat that the chips can be cooled. The system can provide the safe work temperature, besides it suits the chips because of the small size. The paper shows the experiment that we have done to research the performance of small-size vapor compression refrigeration used on chips with high heating flux. We hope to find a solution on heat dissipation of electronic devices.

\section{Variable capacity cooling plate system}

We choose R134a to be the refrigerant. The compressor used in the experiment is MT20D481H whose rotate speed is changeable. The capacity can be changed from $30 \%$ to $100 \%$. 0 to 5 volt signals are sent to 48 volt PWM (Pulse-Width Modulation) control panel with direct current source. By adjusting the PWM impulse frequency of current source, we can control the speed of the compressor motor so as to change the capacity. We record and analyze what influence refrigerant charge brings to suction-exhaust temperature and pressure as well as refrigerating capacity of the system in order to find the best operating state. Figure. 1 is the schematic diagram of variable capacity cooling plate system. We can adjust the way refrigerant flows by control the valves according to what we are going to research. 


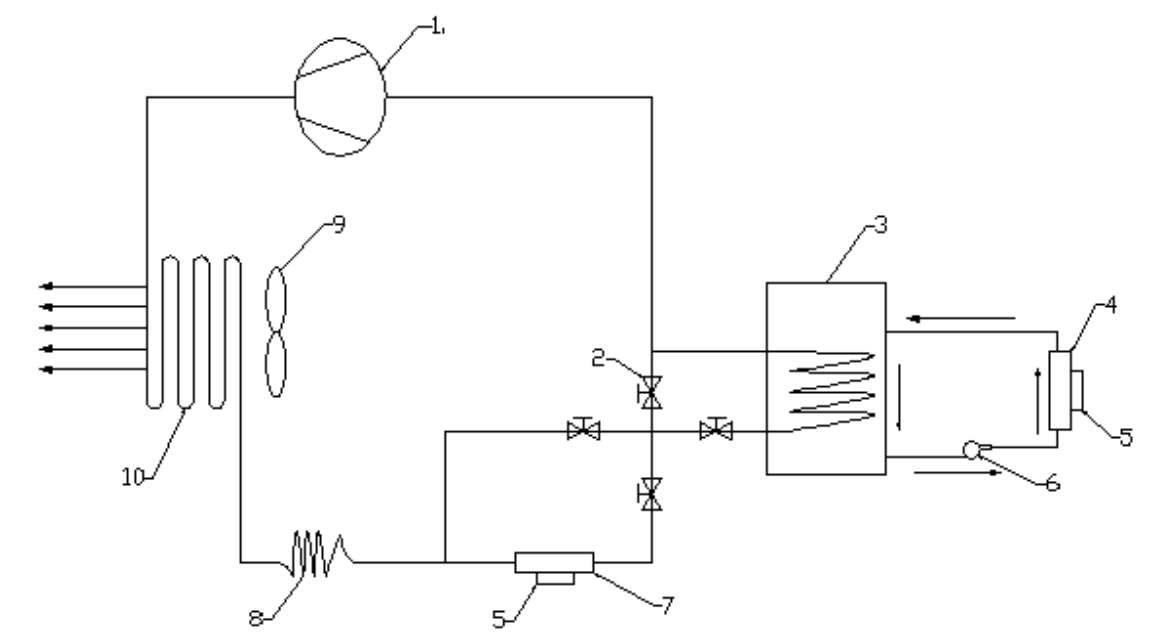

1 :compressor 2:valve 3:water tank 4:water-cooled cooling plate 5:analog chip 6:water pump 7: evaporative cooling plate 8 :capillary 9 :fan 10 :condenser

Figure.1 Variable capacity cooling plate system

\section{Analysis of experiment results for evaporative cooling plate system.}

Influence to suction-exhaust temperature. In order to know the relationship between refrigerant charge and suction-exhaust temperature, we have recorded the changing circumstance when the compressor rotates at the speed of $1800 \mathrm{r} / \mathrm{min}, 3600 \mathrm{r} / \mathrm{min}$ and $4200 \mathrm{r} / \mathrm{min}$ respectively. Figure.2-4 shows the results.

The results show that suction-exhaust temperature declines when refrigerant charge increases within limits. It's known that when the compressor rotates at a certain speed, heat of superheating in the exit of the evaporative cold drawing will decrease as refrigerant charge increases. Compression process of the compressor is assumed to be adiabatic.

The decrease of superheating heat causes the decrease of the suction temperature. We think the pressure ratio is almost invariant, so that the exhaust temperature decreases as well. In addition, when refrigerant charge surpasses 75 kilos, the suction-exhaust temperature is almost stable.

Influence to suction-exhaust pressure. In order to know the relationship between refrigerant charge and suction-exhaust pressure, we have also recorded the changing circumstance when the compressor rotates at the speed of $1800 \mathrm{r} / \mathrm{min}, 3600 \mathrm{r} / \mathrm{min}$ and $4200 \mathrm{r} / \mathrm{min}$ respectively. Figure.5-7 shows the results.

We can see that suction-exhaust pressure increases when refrigerant charge increases within limits. It's known when refrigerant charge increases, compressor suction amount per unit of volume is less than gaseous refrigerant in the cooling plate. So that there will be more gaseous refrigerant in the cooling plate, causing the suction pressure to increase. Meanwhile refrigerant can't pass the capillary in time as the flow of the refrigerant increases, causing the exhaust pressure to increase. We can also find that when refrigerant charge surpasses 75 kilos, the suction-exhaust pressure is almost stable. 


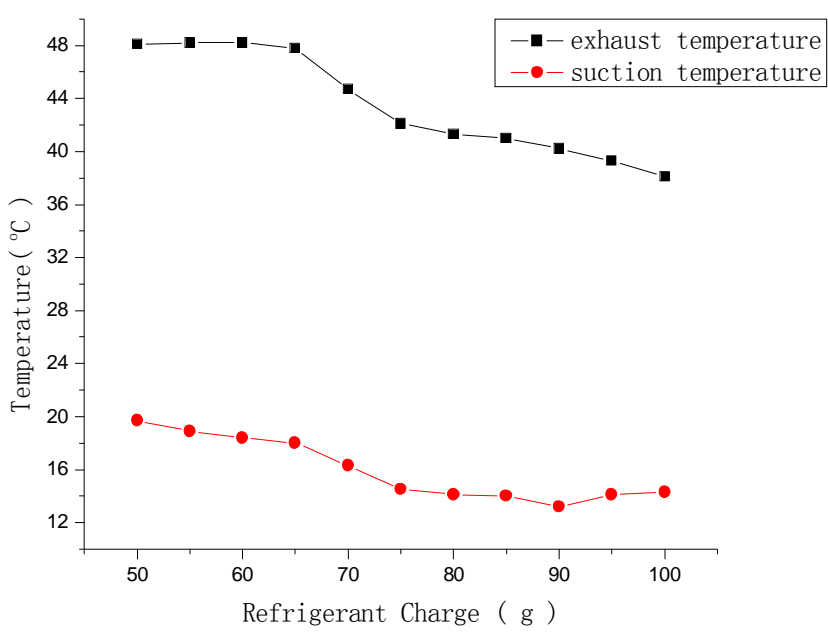

Figure.2The relationship between refrigerant charge and suction-exhaust temperature at the speed of $1800 \mathrm{r} / \mathrm{min}$

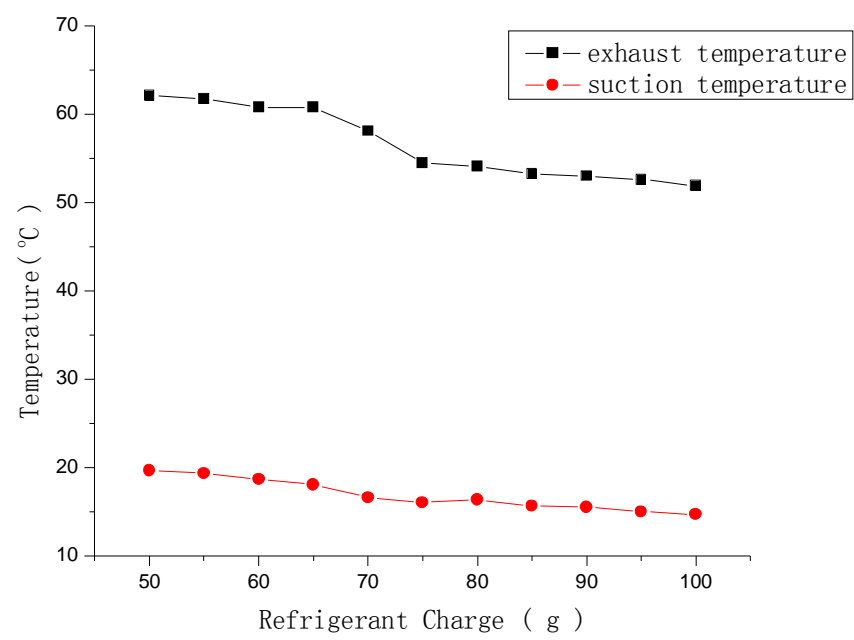

Figure.4The relationship between refrigerant charge and suction-exhaust temperature at the speed of $4200 \mathrm{r} / \mathrm{min}$

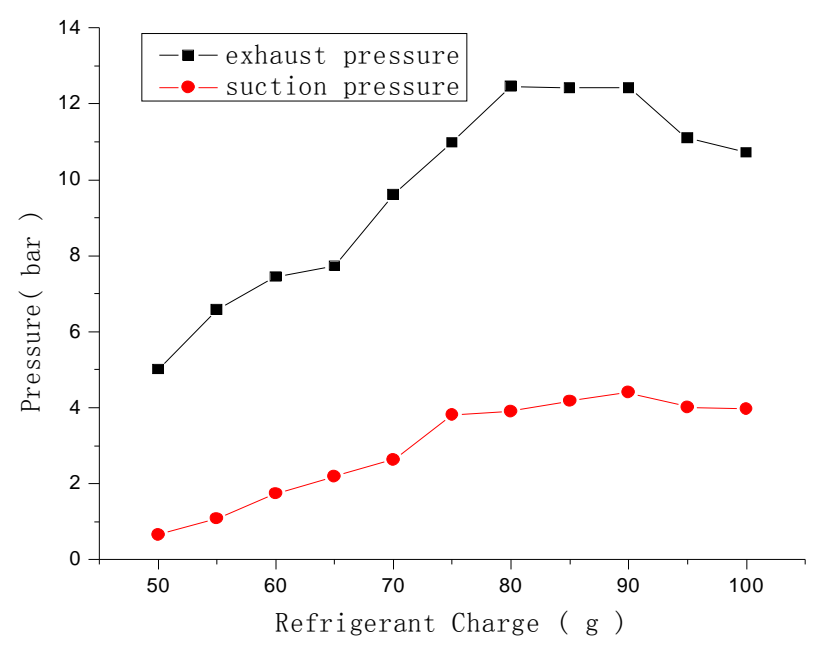

Figure.6The relationship between refrigerant charge and suction-exhaust pressure at the speed of $3600 \mathrm{r} / \mathrm{min}$

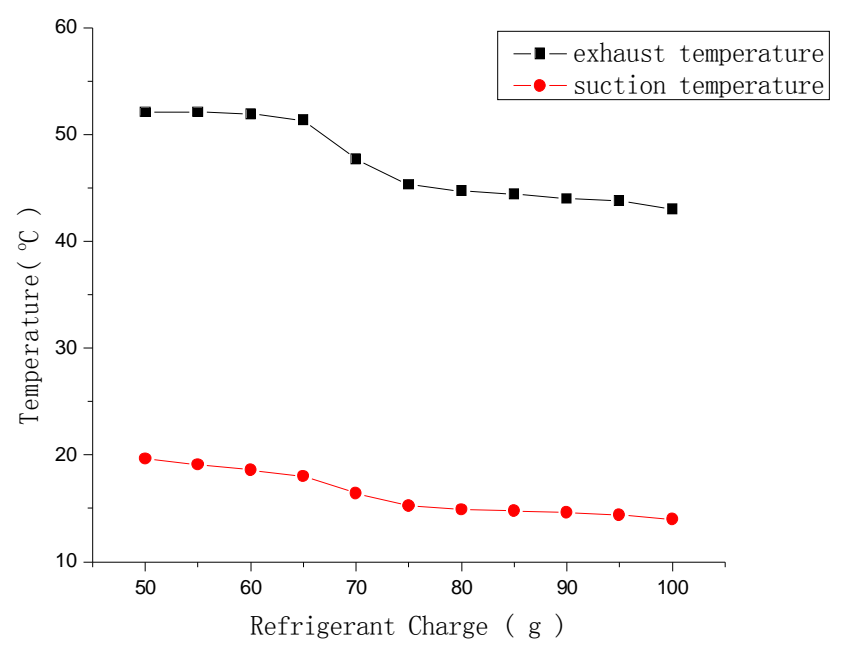

Figure.3The relationship between refrigerant charge and suction-exhaust temperature at the speed of $3600 \mathrm{r} / \mathrm{min}$

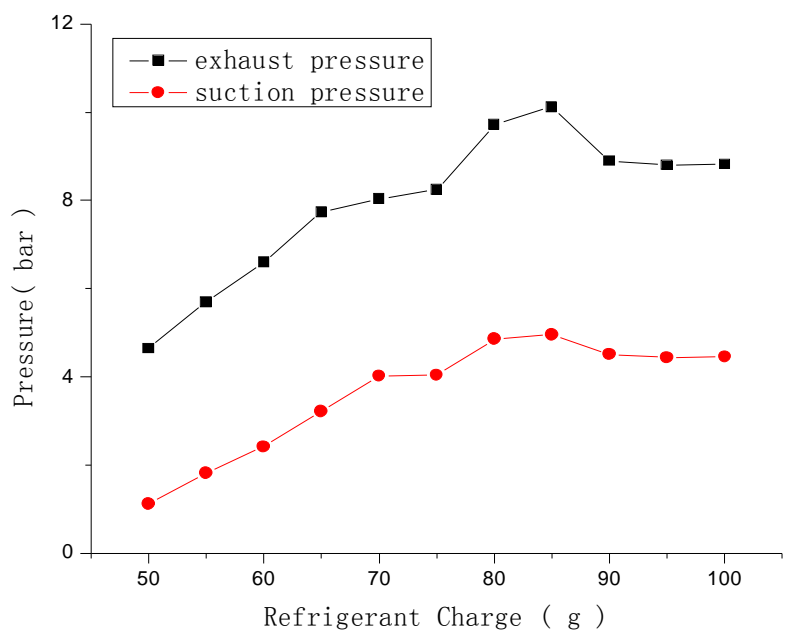

Figure.5The relationship between refrigerant charge and suction-exhaust pressure at the speed of $1800 \mathrm{r} / \mathrm{min}$

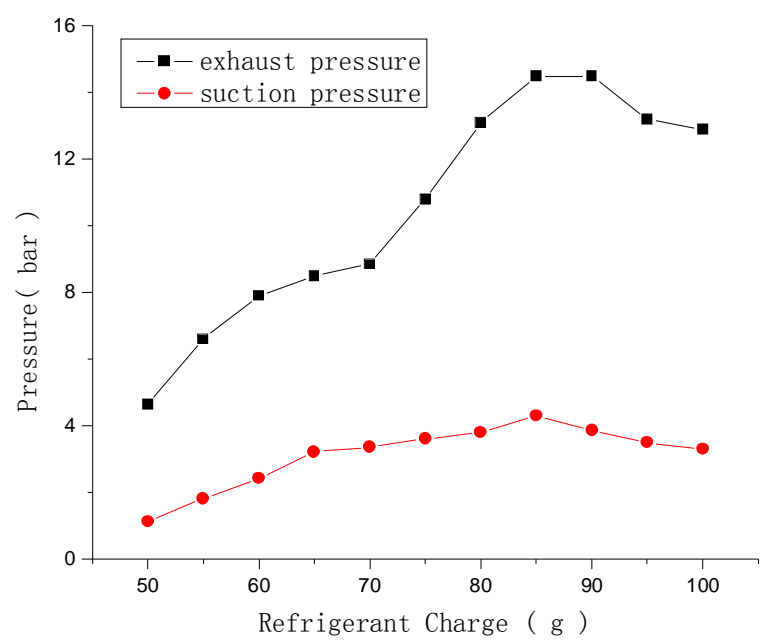

Figure.7The relationship between refrigerant charge and suction-exhaust pressure at the speed of $4200 \mathrm{r} / \mathrm{min}$

Influence to refrigerating capacity of the system. Figure8. is the relationship between refrigerant charge and refrigerating capacity under different compressor rotating rates. At the former state, 
refrigerating capacity keeps increasing along with the increase of refrigerant charge, which makes the effective heat exchange area increase. But, it decreases after reaching a top state. Because evaporating temperature and pressure will rise if refrigerant charge continues being increased. We can see that as for cooling system there exists the most optimal state. To increase or decrease refrigerant charge from this state will effect evaporating and condensing temperature as well as pressure. And then refrigerating capacity of the system will decline [5][6].

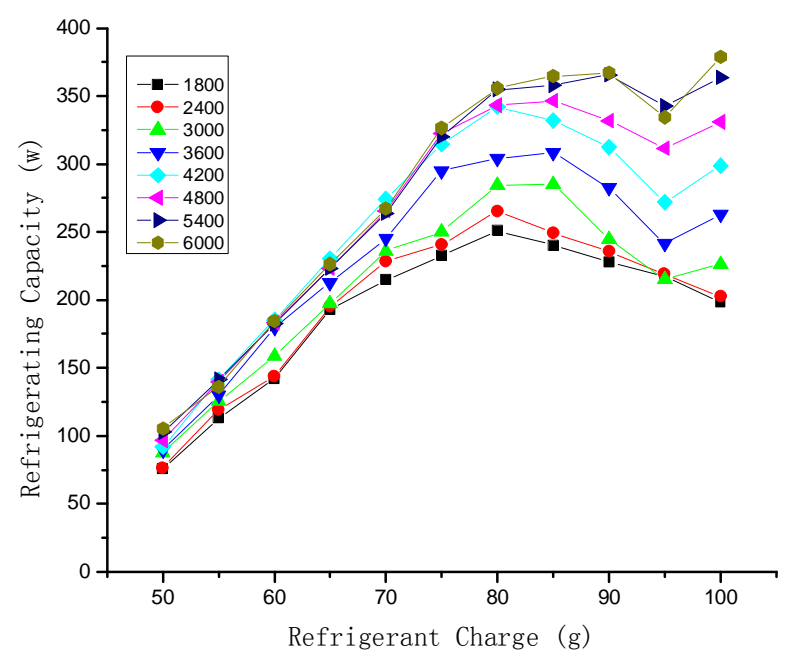

Figure. 8 The relationship between refrigerant charge and refrigerating capacity under different compressor rotating rates.

\section{Contrast of evaporative cooling plate and water-cooled cooling plate}

According to the analysis, we use 70 kilos refrigerant to contrast the cooling ability of evaporative cooling plate and water-cooled cooling plate to cool $100 \mathrm{~W}$ and $200 \mathrm{~W}$ simulative chips respectively.Figure. 9 and Figure. 10 show the results.

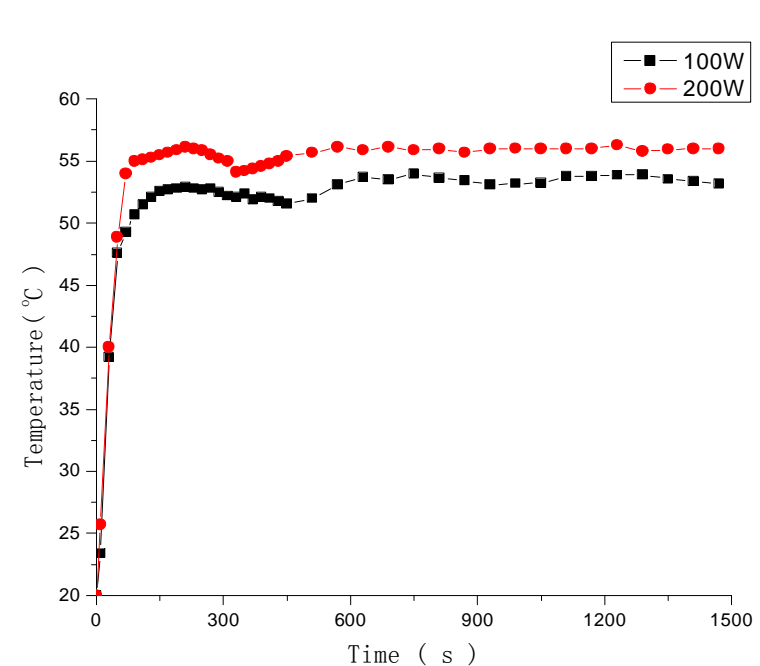

Figure9. The chips cooled by evaporative cooling plate

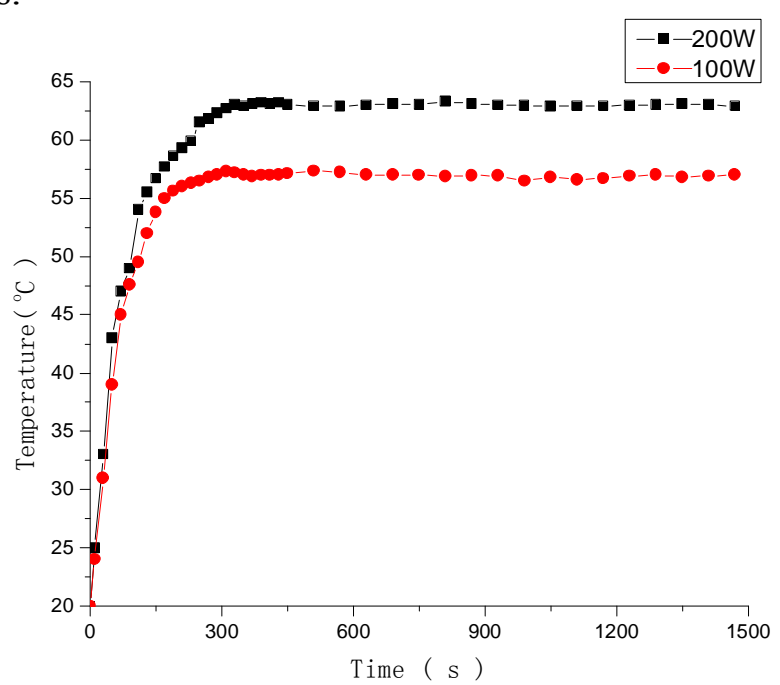

Figure10. The chips cooled by water-cooled cooling plate

We can find that when the power of the chip is $100 \mathrm{~W}$ and $200 \mathrm{~W}$, evaporative cooling plate can keep the temperature under $52^{\circ} \mathrm{C}$ and $56.1^{\circ} \mathrm{C}$ respectively, compared with $57^{\circ} \mathrm{C}$ and $64^{\circ} \mathrm{C}$ cooled by water-cooled cooling plate. They all meet requirements of chips to work safely. But, it shows that the evaporative cooling plate can make the temperature stable more quickly. It takes water-cooled cooling plate almost 5 times time to cool the chip under the same condition. 


\section{Summary}

In this paper, we have researched the performance of the evaporative cooling system used for cooling the chips with high heating flux. With the increase of refrigerant charge, refrigerating capacity of the system increases until it reaches the most optimal state. According to the contrast experiment, it shows that it is more efficient for evaporative cooling plate to cool the chip compared with water-cooled cooling plate. Moreover, it can keep the temperature at a lower state.

But because the energy stored in cooling plate isn't so much, the run state of the cooling system should be kept changing to meet the requirement of heat dissipation. More experiment need to be done to confirm the parameter and variation range, so as to run intelligently as we hoped.

\section{Acknowledgements}

This work was financially supported by the National College Students' Innovative Entrepreneurial Project of China (141025213)

\section{References}

[1] GJB/Z 299C-2006. Electronic Equipment Reliability Prediction Handbook. GAD Military Standard Publish Department(2006)

[2] Zhong Jiang. Application of Copper for Radiator of CPU. Copper Engineering. (2013)7-10

[3] Hong Cao, Qian Lv, Ning Han. Contrast of Thermal Simulation and Test Data of Electronic Equipment Cooled by Forced Convection. Television Technology. 48(2008)109-112

[4] Anlin Zhao, Jianyun Deng, Mingwei Tong. Experimental Research on Performance of R600a in Gravity Assisted Heat Pipe Electronic radiator. The $8^{\text {th }}$ National Heat Pipe Conference . (2002)124-131

[5] Wenbin Wang. Experimental Research on Refrigerant charge of Small-sized Air Cooled Heat Pump. Refrigeration \& Air-Conditioning. 22(2008)114-117

[6] Yuting Wu, Chongfang Ma, Xiaohui Zhong. Development and Experimental Investigation of A Miniature-scale Refrigeration System. Energy Conversion and Management. 51(2010)81-88 